den rohstoffnachfragenden Industrien aufbereitete Informationen zur Verfügung stellen. Informationsaustausch zwischen diesen Wirtschaftsakteuren dürfte zu einer besseren Abstimmung von Rohstoffangebot und -nachfrage führen und die risikobehaftete und damit kostenträchtige Volatilität auf den Rohstoffmärkten reduzieren.

\section{Ausblick}

Rohstoffsicherheit ist kein Selbstzweck, sondern entscheidende Voraussetzung für die Wettbewerbsfähigkeit der Industrie und damit für Wohlstand, Wachstum und Beschäftigung in Deutschland und Europa. Niemand kann die künftige Entwicklung der Rohstoffmärkte voraussagen. Die eingehende Analyse der treibenden Faktoren und die Herstellung von Transparenz über fundamentale Marktdaten erlauben es jedoch, belastbare Entwicklungstrends zu identifizieren. Dieser Trend geht in Richtung einer insgesamt steigenden Rohstoffnachfrage. Der Preisverfall im Zuge der globalen Finanz- und Wirtschaftskrise hat an der grundsätzlichen Bedeutung, unsere Rohstoffabhängigkeit zu reduzieren und sich um eine nachhaltige Sicherung unserer Rohstoffimporte zu bemühen, nichts geändert. Dadurch wird auch der schrittweise Aufbau eines Global-Governance-Systems für Ressourcenmanagement immer dringlicher, das am Eigeninteresse von Unternehmen und Staaten sowie an den aufgeführten internationalen Initiativen anknüpft. ${ }^{19}$

19 Bleischwitz und Bringezu schlagen hierzu drei Eckpfeiler vor: das „International Panel for Sustainable Resource Management“, ein Internationales Abkommen zum nachhaltigen Ressourcenmanagement und eine Internationale Agentur zum nachhaltigen Ressourcenmanagement, ebd. S. 9ff.

\title{
Präventive Ressourcensicherheitspolitik. Initiativen und Anforderungen an ein Global Governance-System
}

\author{
Raimund Bleischwitz*
}

\begin{abstract}
Resources of strategic importance are supposed to take centre stage in future conflicts. On this background, the author argues for the implementation of a preventive resource security policy in form of a multi-level approach that systematically includes non-governmental stakeholders. The main contribution of this article is the identification of some policy approaches and international initiatives relevant for a preventive resource security policy. In conclusion, the crucial importance of a global governance system is emphasized which includes an international agreement on sustainable resource management.
\end{abstract}

Keywords: Rohstoff- und Ressourcensicherheit, Transparenz, Ressourcenproduktivität, internationales Abkommen Security of raw materials and resources, transparency, resources productivity, international agreements

\section{Präventive Ressourcensicherheitspolitik - Rohstoffsicherheit plus Nachhaltigkeit}

Rohstoffsicherheit wird häufig auf den Zugang zu Rohstoffen und ihre kostengünstige Nutzung fokussiert. Eine Studie des US-amerikanischen National Intelligence Council (2008) entwirft ein beunruhigendes Szenario, wonach strategische Rohstoffe, die nur in wenigen Ländern abgebaut oder verarbeitet werden, im Mittelpunkt künftiger Konflikte stehen werden. Diese könnten sowohl bestehende Spannungen umfassen - genannt wird das Verhältnis zwischen China und Indien - als auch neue terroristische Attacken auf verwundbare Industriesysteme.

Derartige Konflikte könnten sich nicht nur bei Erdöl oder Erdgas ergeben. Die Rohstoffwirtschaft in Ländern Zentralafrikas und anderen schwachen Staaten bietet ideale Bedingungen für

Prof. Dr. Raimund Bleischwitz ist stellv. Forschungsgruppenleiter in der Forschungsgruppe Stoffströme und Ressourcenmanagement am Wuppertal Institut für Klima, Umwelt, Energie. schnelle Gewinne: Rohstoffe wie Cassiterit, Coltan, Gold oder Kobalt sind dort auf abenteuerliche Weise leicht zugänglich, erfordern wenig Transportaufwand und erzielen einen hohen Marktpreis. Dies ist nicht allein eine Einladung zu Schattenwirtschaft, illegalen Aktivitäten und lokalen Konflikten. Es kann sich auch eine Konfliktspirale ergeben, in die Nachbarstaaten, Unternehmen und westliche Industrieländer einbezogen sind.

Der folgende Beitrag argumentiert vor diesem Hintergrund für eine präventive Ressourcensicherheitspolitik. Darunter wird ein Mehrebenenansatz verstanden, der vorausschauende Strategien der Ressourcenschonung entwickelt und dabei gezielt nichtstaatliche Akteure aktiviert. Für eine solche Perspektive sprechen folgende Gründe:

Erstens geht es nicht um Rohstoffe als solche, sondern um ihre Funktion für menschliche Bedürfnisbefriedigung und Wohlstand. Die hohen Rohstoffpreise haben einen Paradigmenwechsel eingeleitet, in dessen Folge ein reines Angebotsdenken 
- welche Rohstoffmengen werden benötigt und wie können sie beschafft werden - zugunsten einer Systemperspektive der Ressourcennutzung überwunden wird.

Zweitens sind die Herausforderungen des Klimawandels auch für die Ressourcenpolitik relevant: Eine britische Studie des Resource Efficiency Knowledge Transfer Network (2008) zeigt, dass viele Abbau- und Verarbeitungsgebiete in Gegenden liegen, die von den Folgen der Klimaveränderung betroffen und mithin erhöhten Risiken ausgesetzt sind. Zudem sind neue und saubere Energien wie etwa Windenergie, Hybridantrieb, Photovoltaik, Brennstoffzellen oder Wasserstoffnutzung auf bestimmte Stoffe angewiesen. Die Rohstoffinitiative der EU (EC 2008: 17) bezeichnet etwa 20 Stoffe als kritisch, darunter Antimon, Chrom, Kobalt, Lithium, Platin (PGM), Wolfram und Vanadium. Eine Konsequenz sind verstärke Recyclingbemühungen, d. h. die Frage wo und von wem derartige Rohstoffe wieder aufgearbeitet werden, erhält eine sicherheitspolitische Dimension.

Drittens spricht für eine erweiterte Perspektive, dass die Nutzung von Rohstoffen immer mit Umweltbelastungen verbunden ist. Die Herstellung von Aluminium, Stahl und Zement verursacht gegenwärtig weltweit mehr als zehn Prozent des Treibhausgases CO2; über den gesamten Lebensweg aller Materialien hinweg entstehen Abfälle und Emissionen. Auch die Verlagerung umweltintensiver Produktionsanlagen und der Export fragwürdiger Gebrauchtgüter in Entwicklungsländer sind in diesem Zusammenhang zu nennen. Das Umweltprogramm der Vereinten Nationen (VN) hat deshalb Ende 2007 das Expertengremium „International Panel on Sustainable Resource Management" (IPSRM) eingerichtet, das in Kürze seine ersten Berichte zu den Themen Bio-Kraftstoffe und seltene Metalle vorlegen soll. Der folgende Beitrag nennt einige Politikansätze und internationale Initiativen, die für eine präventive Ressourcensicherheitspolitik relevant sind. Letzten Endes wird für den Aufbau eines Global Governance-Systems plädiert, das auch ein internationales Abkommen zum nachhaltigen Ressourcenmanagement umfassen müsste.

\section{2. Überwindung des „Ressourcenfluchs“ - Makroökonomie und internationale Investitionsabkommen}

In der Vergangenheit konnten rohstoffreiche Entwicklungsländer aus dem Export ihrer Bodenschätze nur selten Vorteile ziehen. Die Anfälligkeit für Korruption und wirtschaftspolitische Verzerrungen, die mit der einseitigen Ausrichtung auf Rohstoffextraktion einhergehen können, ist als „Ressourcenfluch“ bekannt. Dennoch gibt es Länder, die aus ihrem Rohstoffreichtum Nutzen gezogen haben. In den letzten Jahren haben insbesondere Chile und Botswana wirtschaftspolitisch kluge Rohstoffstrategien verfolgt.

Chile profitierte vor der weltweiten Wirtschaftskrise im Jahre 2008 von einem stark gestiegenen Preis für das Hauptexportprodukt Kupfer. In den Jahren 2003 bis 2007 haben sich Chiles Exporterlöse mehr als verdoppelt. Zudem konnte Chile seine Exportabhängigkeit von Kupfer in den letzten Jahren verringern: von 80 Prozent im Jahr 1970 auf ca. 50 Prozent (2007).
Die Besteuerung der Kupferproduktion hat 2005 15,5 Prozent der Regierungseinnahmen erbracht. Wirtschaftspolitisch ist zu beachten, dass Chile inzwischen eine stabile Demokratie ist und sich die Regierung zur klaren Trennung zwischen Staat und Privatsektor bekennt. Zugleich praktiziert Chile eine sozialpolitische Ausrichtung mit öffentlichen Investitionen im Bildungsbereich.

Botswana ist heute eines der wenigen Länder in SubsaharaAfrika, dessen Bevölkerung ein mittleres Einkommensniveau aufweist, während es vor 40 Jahren, so wie heute noch die meisten Nachbarländer, zu den ärmsten Ländern Afrikas gehörte. Hier ist es gelungen, die Einnahmen aus dem Diamantenexport (etwa 50 Prozent des Gesamtexports) positiv für die Entwicklung des Landes zu nutzen. Gründe für den Erfolg sind eine gute Zusammenarbeit von Privatwirtschaft und Staat (der Monopolist in der Diamantenproduktion, das Unternehmen „Debswana“, gehört je zur Hälfte dem Staat und dem Unternehmen De Beers), ein funktionierendes Rechtssystem, das den Schutz des Eigentums gewährleistet, sowie eine verantwortliche Politik der Regierung, die vor allem in den Bildungsbereich investiert.

Ein weiteres Beispiel ist Ghana - einer der wichtigsten Goldexporteure weltweit. Das Land hat sich 2006 im neuen Minerals and Mining Law (Law 703) zum Eigentümer aller mineralischen Ressourcen auf seinem Territorium erklärt und legt eine zehnprozentige Mindestbeteiligung des Staates an allen Bergbauaktivitäten fest, die mit Zustimmung der Unternehmen erhöht werden kann.

Die Erfolge dieser Länder basieren auch auf internationalen Investitionsabkommen, die die Investitionssicherheit mit regionalen Entwicklungsbelangen ausbalancieren. Die Handelsorganisation der Vereinten Nationen UNCTAD hat in diesem Zusammenhang 2004 das Konzept der Flexibilität entwickelt: Flexibilität in den Zielen der Kooperation, im Regelungsumfang, in der Gesamtstruktur und in der Anwendung. Dabei geht es im Wesentlichen darum, einen für alle Beteiligten bindenden und zugleich offenen Vertrag zu formulieren, der die Eigentums- und Nutzungsrechte so definiert, dass Vertragserfüllung auch in einem veränderten Umfeld gewährleistet werden kann. Maßnahmen, um dies sicherzustellen, sind Verknüpfungen zwischen ausländischen Investoren und inländischen Unternehmen, Kapazitätsaufbau und technische Hilfe, die Verringerung informeller Investitionshindernisse, gemeinsame Aktivitäten zur Investitionsförderung, Zugang zu Kapital, finanzielle und steuerliche Anreize und die Schaffung eines institutionellen Mechanismus zur Koordinierung der Aktivitäten. Auch legen neuere Abkommen ein größeres Gewicht auf Anliegen der öffentlichen Politik in Fragen des Gesundheitsschutzes, der Sicherheit und der Umwelt.

Viele Entwicklungsländer konnten in den Jahren hoher Rohstoffnachfrage günstigere Regelungen der Besteuerung gegenüber Unternehmen durchsetzen. Auch dafür sind Verträge geeignet, wenn internationale Bergbauunternehmen z. B. zwischen einem garantierten Steuersatz für einen längeren Zeitraum und flexiblen Steuersätzen mit höheren Risiken wählen können. Am Umsatz bzw. Gewinn orientierte Steuern haben dabei grundsätzlich Vorteile gegenüber festgelegten Lizenzeinnahmen, da sie einen Anreiz zur Ressourcenschonung 
geben und bei etwaigen Knappheitspreisen ebenfalls steigen. Ein schrittweiser Anstieg solcher Ressourcensteuern wäre wirtschafts- und entwicklungspolitisch vernünftig.

\section{Rohstofffonds als Instrument regionaler Konfliktprävention}

Rohstofffonds haben sich in Ländern wie Norwegen bewährt und werden u.a. in Kasachstan eingeführt. Sie stellen für Teile Asiens und Lateinamerikas, insbesondere jedoch für Zentralafrika, eine wichtige Perspektive dar. Dort werden größere Rohstoffreserven vermutet, deren Erschließung mit der begonnenen Demokratisierung neue Entwicklungschancen bieten kann.

Ghana hat beispielsweise 1993 seinen Mineral Development Fund (MDF) eingerichtet. Der Fonds wurde mit dem Ziel geschaffen, (a) spezielle Bergbauprojekte zu fördern, (b) Bergbaufolgeschäden auszugleichen und (c) die Entwicklung in lokalen Gemeinschaften zu fördern. Der MDF erhält 20 Prozent der Lizenzgebühren des Bergbausektors und wird als Hauptinstrument einer sektoralen Industriepolitik genutzt. Insgesamt gibt es nach gegenwärtigem Eindruck jedoch Zweifel, ob der Fonds in Ghana effektiv wirtschaftet und den Regionen die in Aussicht gestellten Entwicklungsvorteile erschließt.

Die Anlage von Erlösen aus dem Bergbau in Rohstofffonds ist für rohstoffreiche Länder eine relevante Perspektive: Die Heimatregionen können Erlöse erzielen. Als Stabilisierungsfonds erlauben sie die Ansammlung von Überschüssen zu Zeiten hoher Rohstoffpreise und ihren Abbau, wenn die Preise fallen und der Sektor einen wirtschaftlichen Abschwung erlebt. Die angesammelten Mittel können zugleich am Kapitalmarkt investiert werden. Die Industrie ist mittelbarer Nutznießer, weil sie zu stabilen Bedingungen zusätzliche Rohstoffe erwerben kann.

Ein Teil der Erlöse sollte über einen Fonds in die Weiterentwicklung von Technologien (Ressourceneffizienz und Umweltschutz), in soziale Dienste (Krankenversorgung, Renten) und Humankapital (Bildung) fließen. Rohstofffonds bieten die Chance, den „Ressourcenfluch“ durch geeignete GovernanceMechanismen zu reduzieren. Erfolgskriterien für Rohstofffonds sind:

- ein unabhängiges Management, d.h. auch eine wirksame öffentliche Kontrolle,

- Transparenz der Einnahmen und Ausgaben sowie Korruptionskontrolle,

- Zweckbindung der Erlöse für eine nachhaltige und diversifizierte Entwicklung.

\section{Transparenzmaßnahmen als nationale und vertikale Konfliktprävention}

Zahlreiche Erfahrungen zeigen, dass Einnahmen aus Rohstoffen nicht zur Diversifizierung und Modernisierung der Wirtschaft genutzt, sondern in Prestigeprojekte investiert werden, direkt auf die Konten korrupter nationaler Eliten fließen oder aber in unverhältnismäßiger Weise ausländischen Rohstoffunternehmen zugutekommen. Nach Angaben der Nichtregierungsorganisation Global Witness sind beispielsweise in Angola zwischen 1997 und 2002 mehr als vier Mrd. US-Dollar an staatlichen Erdöleinnahmen spurlos verschwunden. Für die Demokratische Republik Kongo hat ein VN-Expertenbericht 2008 festgestellt, dass ein nennenswerter Teil von Erlösen aus dem Rohstoffhandel in Waffenkäufe fließt, die regionalen Kriegsherren zugute kommen. Rohstoffe gelten hier als unmittelbare Konfliktursache.

Transparenz von Zahlungsflüssen ist deshalb ein grundlegendes Anliegen vieler internationaler Organisationen. Zahlungsflüsse öffentlich zu machen trägt dazu bei, dass Einnahmen für sinnvolle Zwecke verwendet werden. Transparenz gilt als Voraussetzung für demokratische Kontrolle und damit für die effiziente Allokation öffentlicher Ausgaben. Auf dem G8-Gipfel in Heiligendamm 2007 haben sich die Staats- und Regierungschefs der führenden Industrieländer verpflichtet, auf Unternehmen dahingehend einzuwirken, dass sie Einkommensflüsse und Zahlungsströme offenlegen. „Mehr Transparenz im Grundstoffsektor ist für Rechenschaftspflichtigkeit, verantwortungsbewusstes staatliches Handeln und nachhaltiges Wirtschaftswachstum weltweit von entscheidender Bedeutung“, heißt es in der Abschlusserklärung.

Engagierte Nichtregierungsorganisationen sind z. B. die vom damaligen britischen Premierminister Tony Blair initiierte „Extractive Industries Transparency Initiative“ (EITI) und „Publish What You Pay". Während EITI auf dem Prinzip der Freiwilligkeit basiert und den Regierungen in den Rohstoffländern eine zentrale Rolle bei der Schaffung von Transparenz einräumt, nimmt die „Publish What You Pay“-Initiative stärker die Rohstoffkonzerne und die Industrieländer in die Pflicht. Staaten, die EITI beitreten, verpflichten sich unter anderem,

- regelmäßig alle Zahlungen der Unternehmen im Erdöl-, Erdgas- und Bergbausektor an die Regierung zu melden sowie alle Einnahmen der Regierung aus diesem Industriezweig zu veröffentlichen,

- Zahlungen und Einnahmen von einem unabhängigen Auditor prüfen zu lassen und gegebenenfalls Unstimmigkeiten aufzuklären,

- in die Veröffentlichungspflicht auch die staatlichen Unternehmen des Sektors einzubeziehen,

- einen „Multi-Stakeholder-Prozess“ zu etablieren, in dem die nationalen EITI-Aktivitäten und die veröffentlichten Informationen überprüft werden,

- einen realistischen und finanzierbaren Fahrplan zu erstellen sowie einen nationalen Koordinator zu ernennen und

- in die Veröffentlichungspflicht auch die staatlichen Unternehmen des Sektors einzubeziehen.

Mitte 2008 hatte zwar noch kein Land alle Anforderungen erfüllt, doch 22 Staaten haben bereits Kandidatenstatus inne; dabei fällt die Einbeziehung zahlreicher afrikanischer und zentralasiatischer Staaten ebenso auf wie die Abwesenheit der größeren Schwellenländer. Mehr als 20 Bergbauunternehmen unterstützen die Initiative ebenfalls. Angola wurde - zumindest vorübergehend - aus dem Prozess ausgeschlossen, nachdem es 
Unternehmen, die ihre Zahlungsflüsse offenlegten, mit dem Entzug der Förderrechte bedroht hatte.

Politisch sollten alle Möglichkeiten genutzt werden, damit die Umsetzung der Kriterien gelingt und vor allem die rohstoffreichen Länder und Schwellenländer die Transparenzkriterien des EITI akzeptieren. Wichtig ist dabei auch, die lokale Bevölkerung einzubeziehen. Die Aarhus-Konvention über den Zugang zu Umweltinformationen bietet rechtliche Anhaltspunkte für verbindliche Verpflichtungen.

Politisch wird derzeit debattiert, ob Transparenzinitiativen ausgeweitet und mit schärferen Sanktionsmöglichkeiten versehen werden sollten. Auf den ersten Blick erscheint dies einleuchtend. Dennoch ist das Risiko zu beachten, dass weiche Regeln in Bereiche vordringen, die von der Legitimation her dem Staat vorbehalten sein sollten, und damit ihre eigentliche Stärke - die Attraktivität für viele Beteiligte - verloren geht. Politisch spricht dies für einen zweigleisigen Ansatz, der einerseits Projekte wie EITI unterstützt, andererseits die Formulierung verbindlicher internationaler Regelungen anstrebt.

Mittelfristig sollte eine umfassende Transparenz in den Vertragsbeziehungen zwischen Bergbau, Behörden und Banken sowie in den Wertschöpfungsketten mit der weiterverarbeitenden Industrie und Zuliefererindustrien hergestellt werden. Dabei sollten alle Steuern, Abgaben und Gebühren sowie die Verwendung von Einnahmen in öffentlichen Haushalten transparent gemacht werden. Erfahrungen z.B. aus der Holzwirtschaft in Liberia zeigen, dass es zusätzlich sinnvoll ist, das Konzessionsvergabeverfahren mit einzubeziehen.

\section{Zertifizierung als vertikale Konfliktprävention}

$\mathrm{Zu}$ beachten sind die asymmetrischen Informationen entlang der Wertschöpfungsketten im Rohstoff- und Materialflusssystem. Anders als in der Holzwirtschaft, wo der International Forestry Stewardship Council etabliert ist, und bei Meeresfischen, wo der International Marine Stewardship Council eingerichtet werden konnte, existiert im Bereich Rohstoffe bislang kein Zertifizierungssystem. Da aber Rohstoffe im Verarbeitungsprozess umgewandelt werden und häufig in komplexe Produkte einfließen, ruft dies grundlegende Wissensprobleme hervor: Endkunden wissen oft nicht, dass ein bestimmter Stoff in ihren Produkten enthalten ist.

Asymmetrische Informationen gelten insbesondere zwischen Anbietern von gebrauchten Produkten, die seltene Elemente enthalten, und der Recyclingwirtschaft. Gegenwärtig ist nicht bzw. nur selten zu erkennen, in welcher Qualität diese Materialien vorliegen. Als Folge kommt es zur sogenannten adversen Selektion: Nachfrager glauben, dass es sich um minderwertige Qualität handelt und bieten einen niedrigen Preis. Materialien werden buchstäblich, verramscht'. Der Anreiz zum hochwertigen Recycling ist gering.

Abhilfe könnte eine Zertifizierung für Nutzerketten und Materialien schaffen. Der G8-Gipfel 2007 in Heiligendamm hat den Aufbau zertifizierter Handelsketten beschlossen. Die deutsche Bundesanstalt für Geowissenschaften und Rohstoffe
(BGR) arbeitet seitdem an einem Vorhaben im Bereich Coltan (Tantalerze) - dem Material, dessen Abbau in Zentralafrika laut einer VN-Expertengruppe den Bürgerkrieg mit antreibt und u.a. den Bestand von Gorillas gefährdet. Zertifizierte Handelsketten müssten wegen dieser Zusammenhänge nicht nur geochemisch zuverlässig, sondern zugleich institutionell robust sein. Der Aufbau von zertifizierten Handelsketten ist sicherlich erstrebenswert, muss jedoch mit politischen Lösungen in Zentralafrika und anderen regionalen Konfliktherden einhergehen. Diese politischen Lösungen müssen mindestens eine zuverlässige und öffentliche Kartierung umfassen, wo genau welcher Bergbau betrieben wird und wo welche Vorkommen vermutet werden. Zudem sollte der Waffenhandel stärker auf den Prüfstand gestellt werden.

$\mathrm{Zu}$ beachten ist ferner der gesamte Materialkreislauf. Auch im Bereich Recycling und Entsorgung blüht international die Korruption, werden Steuern hinterzogen und Geldwäsche praktiziert. Mindestanforderung wäre also, dass die Zertifizierung über den gesamten Lebensweg eines Stoffes hinweg aufrechterhalten werden kann. Neue Kennzeichnungssysteme mit einem sogenannten Radio Frequency Identification DeviceSystem (RFID) könnten dabei hilfreich sein. RFIDs werden in unterschiedlichen Kontexten als Instrument zur Schließung von Recylingkreisläufen diskutiert. Produzenten und wichtige Länder müssten sich zur Einführung derartiger Kennzeichnungssysteme verpflichten. Ferner müsste die Zertifizierung mit verbindlichen Mindeststandards für die Qualität der Rückgewinnung der Stoffe und der Recyclingverfahren einhergehen. Für exportierende Unternehmen aus Schwellenländern steigt der Anreiz sich zu beteiligen, wenn diese Standards für den EUBinnenmarkt gelten und Prüfzeichen bei Nichteinhaltung derartiger Standards entzogen werden können.

\section{Trockenlegung von Steueroasen und internati- onale Besteuerung von Kapitaleinnahmen}

In den vergangenen Jahren war es lukrativ, Gewinne aus dem Bergbau an den internationalen Kapitalmärkten zu investieren und dort hohe Zusatzrenditen zu erzielen. Dies hatte den Nachteil, dass zum einen nur wenig in die Nachhaltigkeit des Bergbaus investiert wurde und zum anderen eine unheilvolle Beschleunigung der Finanzströme entstand. Abhilfe könnte ein Vorschlag von Hans-Werner Sinn (2008) schaffen: Trockenlegung der Steueroasen und Einführung einer Quellensteuer auf Kapitalerträge von Besitzern von Rohstoffvorkommen. Dies würde nicht nur die allgemeinen Bestrebungen der internationalen Finanzpolitik unterstützen, sondern zugleich einen Anreiz zur Ressourcenschonung bieten. Eine Quellensteuer auf Kapitalerträge erzeugt starke Anreize, einen größeren Wertanteil des Vermögens unter statt über der Erdoberfläche zu halten. Schnelle Profite - eine der gegenwärtigen Hauptursachen für Ressourcenkonflikte - würden dadurch deutlich erschwert. 


\section{Integrierte Landnutzung für Kleinbergbau und Öko-Tourismus}

In Teilen Zentralafrikas gibt es besondere geologische Bedingungen. Dort sind einige Rohstoffe leicht abbaubar und die Gebiete sind bislang schlecht an Transportrouten angebunden. Dies hat den Kleinbergbau begünstigt. Eine aktuelle Studie von Nicholas Garret und Harrison Mitchel (2009) hebt die soziale Dimension dieser Entwicklung hervor: Bergbau bietet Einkommen und kann für den Aufbau leidlich stabiler Strukturen in fragilen Regionen nutzbar gemacht werden. Diese Perspektive gilt insgesamt für eine integrierte Landnutzung. Den Regionen ist nicht geholfen, wenn die Landwirtschaft in Zeiten hoher Rohstoffpreise vernachlässigt wird. Infolgedessen muss die Verrechtlichung der Landnutzung mit integrierten Nutzungskonzepten verbunden werden, die sowohl Landwirtschaft als auch Bergbau umfassen.

In einigen Regionen kann der Naturschutz Tourismuspotenziale bieten. Eine Studie des Umweltprogramms der Vereinten Nationen (UNEP) zum Gorillatourismus in Uganda zeigt, dass Touristen bereit sind bis zu 500 US-Dollar pro Reise zusätzlich zu zahlen, um Gorillas in ihrer natürlichen Umgebung beobachten zu können (Redmond et al. 2008). Übertragen auf jährliche Besucherzahlen und die Lebenswartung eines Gorillas ergibt sich ein ökonomischer Wert von etwa vier Millionen US-Dollar pro Gorilla - ein Anreiz für den Erhalt der Lebensräume seltener Arten. Insgesamt gibt es also jenseits des Raubbaus durchaus lukrative Möglichkeiten; sie müssten international nur gefördert und eingefordert werden.

\section{Materialeffizienz und Ressourcenproduktivität als ökonomische Erfolgsstrategie}

Ein ganz anderer Ansatzpunkt ergibt sich für die Industrie. Unter Materialeffizienz versteht man die gezielte Effizienzsteigerung des Produktionsfaktors Material. Nach den Arbeiten des Statistischen Bundesamts müsste der Anreiz für die Unternehmen grundsätzlich hoch sein, da die Materialkosten in Deutschland etwa 42 Prozent des Bruttoproduktionswerts der Unternehmen des produzierenden Gewerbes ausmachen. Materialeffizienz und Ressourcenproduktivität werden in einem neuen Handbuch der OECD (2008) auf Basis von lebenszyklusweiten Stoffstromanalysen definiert. Das Handbuch nennt Ressourcen als Oberbegriff und unterscheidet eine

- direkte Materialproduktivität - BIP / direkter Material-Input, d.h. die inländische Rohstoffentnahme plus die Menge der Importe - von einer

- globalen Materialproduktivität - BIP / globaler Materialaufwand, d.h. die genannte Materialmenge plus vorgelagerte Stoffströme im In- und Ausland.

Eine Erhöhung der inländischen Ressourcenproduktivität reduziert die Importkosten, senkt die Rohstoffabhängigkeiten und fördert Kapitaleinsatz in der inländischen Wertschöpfung. Auf der betrieblichen Ebene senkt eine erhöhte Materialeffizienz die Einkaufs- und Nutzungskosten für Material; dies verbessert einen wichtigen Parameter für die Wettbewerbsfähigkeit und erlaubt Ausgaben für Weiterqualifizierung und Innovationen. Zugleich steigt der Spielraum für Lohnerhöhungen und Arbeitsplatzsicherung. Eine an der Universität Osnabrück durchgeführte Modellberechnung ergab einen Nettobeschäftigungsanstieg von 750000 Arbeitsplätzen in Deutschland.

Das Interesse von Unternehmen und der Industrie an einer Erhöhung der Materialeffizienz ist besonders hoch, wenn Materialkosten abgesenkt werden können. Prioritär werden Prozessinnovationen angestrebt, d. h. Fertigungsabläufe optimiert, Verluste bei der Nutzung von Material minimiert sowie der Einsatz von Wasser, Reinigungsmitteln und anderen Betriebsstoffen durch Mehrfachnutzungen verbessert. Eine Studie schätzt ausgewählte Innovationspotenziale in Deutschland wie folgt $\mathrm{ab}$ :

Tabelle 1: Materialeinsatz und Einsparpotenziale der nächsten 7 bis 10 Jahre in vier ausgewählten Branchen

\begin{tabular}{|l|l|l|}
\hline Branche & $\begin{array}{l}\text { Material- } \\
\text { einsatz } \\
\text { (in Mrd. €) }\end{array}$ & $\begin{array}{l}\text { Ge- } \\
\text { schätztes } \\
\text { Einspar- } \\
\text { potenzial } \\
\text { (in Mrd. €) }\end{array}$ \\
\hline $\begin{array}{l}\text { Herstellung von Metallerzeugnis- } \\
\text { sen }\end{array}$ & 18,6 & $0,8-1,5$ \\
\hline $\begin{array}{l}\text { Herstellung von Anlagen zur Elek- } \\
\text { trizitätserzeugung, -verteilung u. ä. }\end{array}$ & 10,2 & $1,5-3,0$ \\
\hline $\begin{array}{l}\text { Chemische Industrie (ohne } \\
\text { Grundstoffindustrie) }\end{array}$ & 11,1 & $1,8-3,4$ \\
\hline Herstellung von Kunststoffwaren & 10,8 & $1,0-2,0$ \\
\hline
\end{tabular}

Quelle: Arthur D. Little/Wuppertal Institut/FHG ISI (2005): Studie zur Konzeption eines Programms für die Steigerung der Materialeffizienz in mittelständischen Unternehmen, Abschlussbericht für das BMWA, Berlin, S. 7.

Unternehmen können die Materialeffizienz und Ressourcenproduktivität in allen Ländern erhöhen, auch in Schwellenund Entwicklungsländern. In der Regel gehen Material- und Energieeinsparungen Hand in Hand. Eine Kostenanalyse sollte nicht allein innerhalb eines Unternehmens durchgeführt werden, sondern über den Lebenszyklus der produzierten Güter hinweg und entlang einer Wertschöpfungskette. International unterstützt der Marrakesch-Prozess diese Bemühungen. Die japanische G8-Initiative zu „3R“ (Reduction - Reuse - Recycling) versucht von der Abfallseite her das Ressourcenmanagement in Ostasien zu verbessern. China hat ein ehrgeiziges Programm zur Kreislaufwirtschaft (Circular Economy) aufgelegt und sucht nach Partnern für die Finanzierung und für technologisches Know-How; mittelfristig steht dabei der Aufbau offener Recyclingmärkte mit Nachhaltigkeitsregeln auf der Tagesordnung. Impulsprogramme zur Förderung von Materialeffizienz und Ressourcenproduktivität können die Informationsbasis vor allem bei kleinen und mittelständischen Unternehmen verbessern und Markteinführungen erleichtern.

Eine am Wuppertal Institut durchgeführte Korrelationsanalyse ergibt einen positiven Zusammenhang zwischen der Wettbewerbsfähigkeit einer Volkswirtschaft und ihrer Ressourcenproduktivität $\left(\mathrm{r}^{2}=0,30, \mathrm{t}\right.$-Statistik und F-Statistik sind im 95 
Prozent Signifikanzbereich). Datengrundlage dieser Analyse sind ein Ländervergleich des Index zur Wettbewerbsfähigkeit des Weltwirtschaftsforums sowie die internationalen Daten zur Ressourcenproduktivität, die auf Basis des direkten Materialverbrauchs und des BIP in US-Dollar und der Kaufkraftparität im Jahr 2000 erstellt wurden. Finnland und einige skandinavische Länder mit hoher Wettbewerbsfähigkeit und unterdurchschnittlicher Ressourcenproduktivität sind im Ländervergleich die Ausnahmen.

Eine Erhöhung der Ressourcenproduktivität kann als präventive Strategie zur Vorbeugung von Rohstoffkonflikten angesehen werden. Sie ist jedoch zu allgemein ausgerichtet, um aktuelle und künftige spezifische Konflikte lösen zu können. Wer die Materialeffizienz in seinem Unternehmen optimieren will, wird die Kosten der Materialnutzung einbeziehen. Dies führt zu Prozessoptimierungen und möglicherweise sogar zu Produkterneuerungen. Für eine strategische Neuorientierung im Hinblick auf die Einbeziehung ökologischer ,Rucksäcke', auf eine Risikominimierung des Bezugs von kritischen Metallen, auf die Entwicklung radikal neuer Produkte, Dienstleistungen und Systeminnovationen sowie auf ein neues Portfolio, in dem die Schwergewichte aussortiert werden, werden jedoch neue Kooperationen und Anreizstrukturen erforderlich sein. Aus diesen Gründen ist eine politische Flankierung unerlässlich. Die Bereitstellung einer oben genannten Landkarte von lokalen und regionalen Rohstoffkonflikten, einer Liste von kritischen Metallen und einer international zugängliche Datenbank zu Stoffströmen sind deshalb notwendige öffentliche Aufgaben.

\section{Global Governance: ein internationales Ab- kommen für nachhaltiges Ressourcenmanage- ment}

Längerfristig sollte die internationale Politik durch ein Abkommen zum nachhaltigen Ressourcenmanagement auf eine solide rechtliche Basis gestellt werden. Ein solches internationales Abkommen diente einem nachhaltigen und friedlichen Ressourcenmanagement sowie Prinzipien von Materialeffizienz und Ressourcenschonung für kritische Rohstoffe. Weitere Ziele sollten in der Entkoppelung des Wohlstands von Ressourcennutzung und Umweltbelastungen liegen sowie in der rechtlichen Verankerung von Standards für Bergbau, Recycling und Entsorgung. Angesichts der Nutzungskonflikte mit Agrarrohstoffen ist zu überlegen, ob man eine nachhaltige Landnutzung mit in das Abkommen aufnehmen soll bzw. wie diese zu verankern wäre.

Ein internationales Abkommen sollte zwei grundlegende Rechtsprinzipien verbindlich verankern:

1) Das Prinzip des „gemeinsamen Erbes der Menschheit“ (common heritage of mankind), wonach Ressourcen einzelnen Staaten und Akteuren zur Nutzung überlassen sind, letzten Endes jedoch in ihrem Gesamtbestand als Erbe der Menschheit angesehen und zumindest in ihren wichtigsten Funktionen an künftige Generationen weitergegeben werden sollten.

2) Das Prinzip der „Materialverantwortung“ (materials stewardship), wonach eine optimale und angemessene
Förderung, Produktion und Nutzung von Rohstoffen zum Wohle der Gesellschaft unter Wahrung von Umweltbelangen stattfinden soll.

Ein Abkommen sollte die Informationsbasis verbessern, Anreize für Lernprozesse schaffen und die Transparenz und den verbesserten Zugang zu ressourcenbezogenen Informationen verbindlich regeln. Der OECD-Bericht zu Stoffstromanalysen von 2008 weist dabei den Weg. Um den Zugang verschiedener Nutzer zu erleichtern, bietet sich die Einrichtung einer öffentlich zugänglichen internationalen Datenbank an. Das Abkommen sollte zudem vorhandene Initiativen sowie die Einführung und Umsetzung von zertifizierten Handelsketten unterstützen und stärken.

Ein langfristig interessanter Ansatz kann eine global koordinierte Ressourcensteuer sein. Eine solche Steuer würde konkret bei der Nutzung (nicht bei Besitz) natürlicher Ressourcen erhoben, ihre Dividende als Unterstützung für den Aufbau in Krisenregionen und die Bedürftigsten verteilt. Mit dieser Perspektive sollte es möglich sein, Nutzungsentgelte auf international gehandelte Rohstoffe und beim nationalen Verbrauch in Industrieländern zu erheben sowie Ziele und Fahrpläne für die Ressourcenschonung zu vereinbaren. Nutzungsentgelte für Flugzeug-Kerosin und Diesel für die internationale Seeschifffahrt sowie für Flughäfen und Häfen sind nützliche Schritte in diese Richtung, für die die internationale Klimapolitik gute Argumente bietet. Ebenfalls machbar könnten Vereinbarungen über ökonomische Anreize in Form von handelbaren Zertifikaten und Gutschriften für Engagement in Entwicklungsländern sein.

Institutionell ist zusätzlich eine internationale Agentur zum nachhaltigen Ressourcenmanagement notwendig, um Informations-, Qualifikations- und Umsetzungsaufgaben dauerhaft wahrnehmen zu können. Die folgende Abbildung zeigt die institutionellen Vorschläge zum nachhaltigen Ressourcenmanagement.

Abbildung 1: institutionelle Vorschläge zum nachhaltigen Ressourcenmanagement

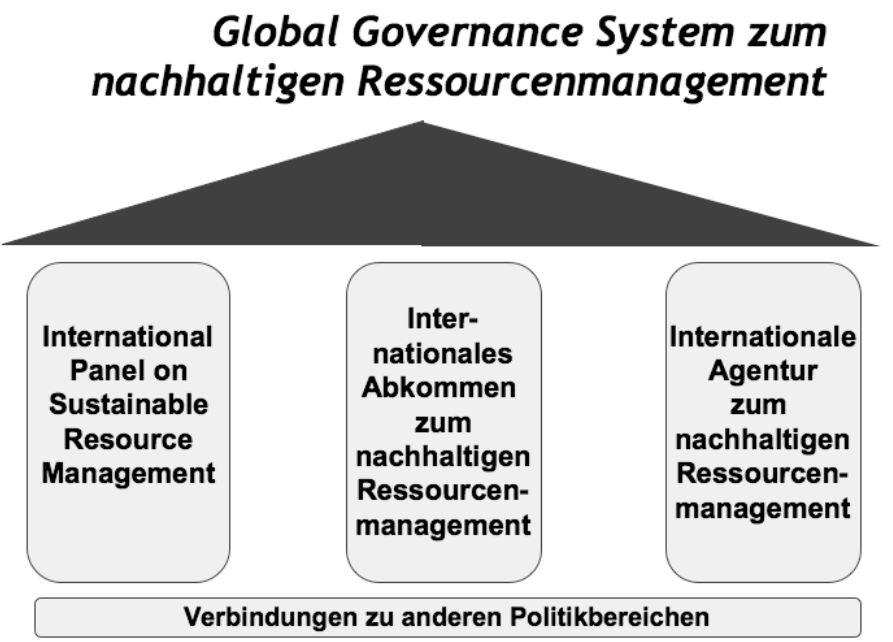

Quelle: Bleischwitz, R. / Bringezu, S. (2007): Globales Ressourcenmanagement - Konfliktpotenziale und Grundzüge eines Global Governance-Systems, SEF Policy Paper No. 27, Foundation Development and Peace (SEF), Bonn. 


\section{Ausblick}

Präventive Sicherheitspolitik wurde in diesem Beitrag als Mehrebenenansatz vorgestellt, der eine gute Regierungsführung in Entwicklungsländern, internationale Initiativen, Managementansätze und ein internationales Abkommen umfasst. Damit wird zum Ausdruck gebracht, dass die Politik gefordert ist, eine stärkere Integration von Umwelt-, Wirtschafts- und Technologiepolitik zu betreiben. Die Chancen der Wirtschaft, durch Ressourceneffizienz einen neuen Innovationsmotor anzuwerfen, können diese Bestrebungen zusätzlich motivieren. Dabei sollten jedoch nicht die Augen davor verschlossen werden, dass zum einen akute Krisenregelungsmechanismen erforderlich sind (z. B. in der DR Kongo) und zum anderen die internationale Sicherheitsarchitektur umfassend auf dieses neue Schlüsselthema einzustellen ist. Etwaige Reformen der Vereinten Nationen hat dieser Beitrag noch nicht thematisiert, ebenso wenig den Bezug zum Konzept der menschlichen Sicherheit. Die hier genannten Strategien wirtschaftspolitischer Reformen, der Transparenz und der Einführung eines internationalen Abkommens sind jedoch konkrete Beiträge und Bausteine für eine präventive Sicherheitspolitik.

\section{Literatur:}

Bleischwitz, Raimund und Florian Pfeil, F. (Hgs.) (2009): Globale Rohstoffpolitik - Herausforderungen für Sicherheit, Entwicklung und Umwelt, Reihe EINE WELT Bd. 23, Baden-Baden.
European Commission (2008): The Raw Material Initiative - Meeting our Critical Needs for Growth and Jobs in Europe; Commission Staff Working Document accompanying the Communication from the Commission to the European Parliament and the Council; \{COM(2008) 699\}, SEC(2008) 2741; Brussels, o.J.

Garrett, Nicholas und Harrison Mitchel, (2009): Trading Conflict for Development. Utilising the Trade in Minerals from Eastern DR Congo for Development, Resource Consulting Service London.

National Intelligence Council (2008): Global Trends 2025: A Transformed World, Washington D.C.

Resource Efficiency Knowledge Transfer Network (2008): Material Security - Ensuring resource availability for the UK economy, Chester.

Sinn, Hans-Werner (2008): Das grüne Paradoxon: Warum man das Angebot bei der Klimapolitik nicht vergessen darf, ifo-Institut München, working paper No. 54, München.

Redmond, Ian, Mapesa, Moses und Aggrey Rwetsiba (2008): Forests: More than just trees, United Nations Environment Programme, Nairobi.

United Nations Security Council (2008): Final report of the Group of Experts on the Democratic Republic of the Congo, No. S/2008/773, New York, 12. Dezember 2008.

\title{
'What's Mine is Mine, What's Yours is Negotiable': Self- Sufficiency versus Interdependence in Energy Strategy
}

\author{
Alyson J. K. Bailes*
}

Abstract: Energy security presents several paradoxes including the fact that owning large energy resources may be a source of instability, while a properly managed interdependence of producer and consumer can prove stabilizing. Oil and gas wealth is already known to be linked to failings in economic development and security. Countries that seek to maximize native production from nuclear power face physical and proliferation risks, while large-scale renewable projects raise their own issues of safety and civil freedom. Interdependence based on producers' comparative advantages makes more economic sense and may also force partners to overcome international problems that would otherwise fester into something worse.

Keywords: Energiesicherheit, Erneuerbare Ressourcen, Autarkie, Abhängigkeit

Energy security, renewable resources, autarchy, dependence

\section{Introduction and Intent}

In economics, and in international relations generally, possession of natural assets would normally be considered an advantage compared to the lack of them. This ought to be especially true in the case of native sources of energy (oil, gas, coal, nuclear or renewable), given their importance both for national strength - including the operation of armed forces - and as a trading commodity.

\footnotetext{
* Prof. Alyson Bailes teaches on general security topics and on Nordic and European security at the University of Iceland in Reykjavik.
} 